\title{
Verpacamides A-D, a Sequence of C11 N5 Diketopiperazines Relating Cyclo(Pro-Pro) to Cyclo(Pro-Arg), from the Marine Sponge Axinella vaceleti: Possible Biogenetic Precursors of Pyrrole-2-Aminoimidazole Alkaloids.
}

Carine Vergne, ${ }^{a}$ Nicole Boury-Esnault, ${ }^{b}$ Thierry Perez, ${ }^{b}$ MarieThérèse Martin, ${ }^{a}$ Marie-Thérèse Adeline, ${ }^{a}$ Elise Tran Huu Dau, ${ }^{a}$ Ali Al-Mourabit ${ }^{\star a}$

${ }^{a}$ Institut de Chimie des Substances Naturelles du CNRS, Avenue de la Terrasse, 91198 Gif-sur-Yvette, France

${ }^{b}$ Centre d'Océanologie de Marseille, CNRS UMR-6540, Station Marine d'Endoume, 13007 Marseille, France

\section{General Experiment Procedures}

NMR spectra were recorded on Spectrometers Avance 500MHz Brucker, DRX 600MHz Brucker, Avance 800MHz Brucker and AMX 400MHz Brucker for NOESY experiments. Chemical shifts (expressed in ppm) of ${ }^{1} \mathrm{H}$ and ${ }^{13} \mathrm{C}$ NMR spectra were referenced to the solvent peaks $\delta_{H} 3.31$ and $\delta_{C} 49.1$ for $\mathrm{CD}_{3} \mathrm{OD}, \delta_{\mathrm{H}} 2.50$ and $\delta_{\mathrm{C}} 39.5$ for DMSO$\mathrm{d} 6$, trace of dioxane $\delta_{\mathrm{C}} 66.7$ for ${ }^{13} \mathrm{C}$ in $\mathrm{D}_{2} \mathrm{O}$. HRMS mass spectra were obtained with an electrospray source (Lockspray) coupled with a time of flight analyzer (LCT, Micromass). Samples were prepared in methanol and injected in the MS system using a Waters 2795 system. The mobile phase was $\mathrm{MeOH} /$ water $(50 / 50, \mathrm{v} / \mathrm{v})$ with a $0.2 \mathrm{ml} / \mathrm{min}$ flow. Preparative HPLC (column HyperCarb $5 \mu 150 \times 10 \mathrm{~mm}$ ) were performed on an autoprep system (Waters 600 controller and Waters 600 pump with a Waters 996 PhotoDiode Array detector). Samples were injected by the Waters 2700 Sample Manager. Optical rotations were measured on a Jasco P-1010 polarimeter in $\mathrm{MeOH}$ or $\mathrm{H}_{2} \mathrm{O}$. IR spectra were acquired (neat) using a Perkin Elmer Spectrometer BX FT-IR system.

\section{Animal Material}

The Mediterranean Sponge Axinella vaceleti (Family Axinellidae) was collected by scuba at a depth of $8 \mathrm{~m}$ at Maïre island ('Grotte à Corail') during April 2003. A second sample was collected at a depth of $12 \mathrm{~m}$ at Riou Archipelago ('Grotte de Plane') in April and July 2004. The specimens were immediately frozen at $-80^{\circ} \mathrm{C}$ and lyophilized. Voucher samples are available at the Marine Station d'Endoume, Marseille, France. 


\section{Extraction and Isolation}

The freeze-dried two samples of the Sponge Axinella vaceleti were extracted separately.

Sample one (12 g) was homogenized and extracted with $\mathrm{MeOH}(165 \mathrm{~mL}+2 \times 50 \mathrm{~mL})$ to give $3.70 \mathrm{~g}$ of a crude which was purified by chromatography on silica gel using a gradient of solvent $\left(\mathrm{CH}_{2} \mathrm{Cl}_{2} / \mathrm{MeOH} 100 / 0\right.$ to $\left.0 / 100\right)$. Fractions eluted with $\mathrm{CH}_{2} \mathrm{Cl}_{2} / \mathrm{MeOH}: 80 / 20$ were combined, concentrated (138 $\mathrm{mg}$ ) and purified by preparative thin layer chromatography $\left(\mathrm{CH}_{2} \mathrm{Cl}_{2} / \mathrm{MeOH}: 80 / 20\right)$ to give $5 \mathrm{mg}$ of a nearly pure product. After final purification by $\mathrm{HPLC}\left(\mathrm{H}_{2} \mathrm{O}+0.1 \% \mathrm{HCOOH} / \mathrm{MeCN}+0.1 \% \mathrm{HCOOH}, 90 / 10\right.$ during $7 \mathrm{~min}$ and then gradient to $80 / 20$ in $10 \mathrm{~min}$, flow rate : $4.7 \mathrm{ml} / \mathrm{min}$, UV detection at $195 \mathrm{~nm}$ ), $1.5 \mathrm{mg}$ of compound 6 was obtained.

Fractions eluted with $\mathrm{CH}_{2} \mathrm{Cl}_{2} / \mathrm{MeOH}: 85 / 15$ were combined to give $258 \mathrm{mg}$ of a mixture that was treated by silica preparative thin layer chromatography $\left(\mathrm{CH}_{2} \mathrm{Cl}_{2} / \mathrm{MeOH}: 80 / 20\right)$. A polar mixture (16 mg) containing silica was obtained. The latter was chromatographed on a Sephadex $\mathrm{LH}-20$ column using $\mathrm{MeOH}$ and then finally purified by $\mathrm{HPLC}\left(\mathrm{H}_{2} \mathrm{O}+0.1 \% \mathrm{HCOOH} / \mathrm{MeCN}+0.1 \% \mathrm{HCOOH} 98 / 2\right.$, flow rate: $4.7 \mathrm{ml} / \mathrm{min}$, UV detection TIC) to give $1.2 \mathrm{mg}$ of 9 .

The second sample $(16.2 \mathrm{~g})$ was extracted following the same procedure as for sample one. The crude extract $(4.03 \mathrm{~g})$ was purified by chromatography on silica using a Versa Pack system (silica cartridge 40x150 mm) using $\left(\mathrm{CH}_{2} \mathrm{Cl}_{2} / \mathrm{MeOH}: 100 / 0\right.$ to 0/100) gradient. Fractions obtained by the eluent $\mathrm{CH}_{2} \mathrm{Cl}_{2} / \mathrm{MeOH}: 85 / 15$ gave $158 \mathrm{mg}$ of a mixture that was finally purified by HPLC $\left(\mathrm{H}_{2} \mathrm{O}+0.1 \% \mathrm{HCOOH} / \mathrm{MeCN}+0.1 \% \mathrm{HCOOH} 87 / 13\right.$, flow rate: 4.7 $\mathrm{ml} / \mathrm{min}$, UV detection $254 \mathrm{~nm}$ ) to give $11.3 \mathrm{mg}$ of compound 8. Fractions obtained by the eluent $\mathrm{CH}_{2} \mathrm{Cl}_{2} / \mathrm{MeOH}$ $60 / 40$ gave $169 \mathrm{mg}$ that were purified on silica $\left(\left(\mathrm{CH}_{3}\right)_{2} \mathrm{CO} / \mathrm{EtOAc} / \mathrm{HCOOH} / \mathrm{H}_{2} \mathrm{O} \quad 5 / 3 / 1 / 1\right)$ to provide $4.5 \mathrm{mg}$ of compound 7.<smiles>N=C(N)NCCC[C@H]1NC(=O)[C@@H]2CCCN2C1=O</smiles>

6

Verpacamide A (as formic salt) (6) : All analytical data were identical to the synthetic reported compound. ${ }^{5}$ (see ${ }^{1} \mathrm{H}$ NMR spectra of natural and synthetic 6 ) 


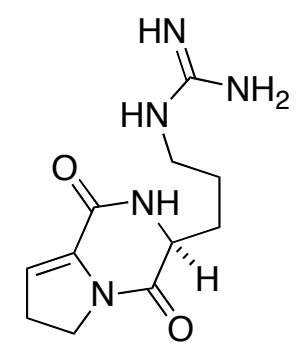

7

Verpacamide B (as formic acid salt) (7) : colorless oil ; $[\alpha]^{24}{ }_{D}=-5.7^{\circ}(c=0.32, \mathrm{MeOH}) ;{ }^{1} \mathrm{H}$ NMR data $\left(\mathrm{CD}_{3} \mathrm{OD}, 500 \mathrm{MHz}\right) \delta_{\mathrm{H}} 6.19(\mathrm{~m}, 1 \mathrm{H}, 9-\mathrm{H}), 4.28(\mathrm{~m}, 1 \mathrm{H}, 4-\mathrm{H}), 4.00(\mathrm{t}, \mathrm{J}=9.0 \mathrm{~Hz}, 2 \mathrm{H}, 7-\mathrm{H}), 3.19$ (t, $J=6.5 \mathrm{~Hz}, 2 \mathrm{H}, 1-$ $\mathrm{H}), 2.80$ (brt, $2 \mathrm{H}, 8-\mathrm{H}), 1.98-1,84(\mathrm{~m}, 1 \mathrm{H}, 3 \mathrm{a}-\mathrm{H}$ and $3 \mathrm{~b}-\mathrm{H}), 1.66-1,55(\mathrm{~m}, 1 \mathrm{H}, 2 \mathrm{a}-\mathrm{H}$ and $2 \mathrm{~b}-\mathrm{H}) ;{ }^{13} \mathrm{C}$ NMR data $\left(\mathrm{CD}_{3} \mathrm{OD}, 125 \mathrm{MHz}\right) \delta_{\mathrm{C}} 164.8$ (C-5), 158.8 (C-14), 134.2 (C-10), 121.5 (C-9), 57.9 (C-4), 46.7 (C-7), 42.3 (C-1), 32.5 (C-3), 28.8 (C-8), 25.2 (C-2) ; IR (neat) 3347, 1666, 1633, 1582 cm ; HRMS (ESI) m/z 252.1453 (252.1461 calcd. for $\mathrm{C}_{11} \mathrm{H}_{18} \mathrm{~N}_{5} \mathrm{O}_{2}, \mathrm{M}+\mathrm{H}$ ).<smiles>N=C(N)N[C@@H]1CC[C@H]2C(=O)N3CCC=C3C(=O)N12</smiles>

8

Verpacamide $\mathbf{C}$ (as formic acid salt) (8) : colorless solid; $[\alpha]^{24}{ }_{D}=+17.4^{\circ}\left(\mathrm{C}=0.15, \mathrm{H}_{2} \mathrm{O}\right) ;{ }^{1} \mathrm{H}$ NMR data $\left(\mathrm{D}_{2} \mathrm{O}, 500 \mathrm{MHz}\right) \delta_{\mathrm{H}} 6.35(\mathrm{t}, J=3 \mathrm{~Hz}, 1 \mathrm{H}, 9-\mathrm{H}), 5.75(\mathrm{dd}, J=4.8$ and $8.1 \mathrm{~Hz}, 1 \mathrm{H}, 1-\mathrm{H}), 4.54(\mathrm{dd}, J=6.8$ and $10.6 \mathrm{~Hz}$, $1 \mathrm{H}, 4-\mathrm{H}), 4.00-3,89(\mathrm{~m}, 2 \mathrm{H}, 7 \mathrm{a}-\mathrm{H}$ and $7 \mathrm{~b}-\mathrm{H}), 2.80(\mathrm{~m}, 2 \mathrm{H}, 8 \mathrm{a}-\mathrm{H}$ and $8 \mathrm{~b}-\mathrm{H}), 2.53(\mathrm{~m}, 1 \mathrm{H}, 2 \mathrm{a}-\mathrm{H}), 2.40(\mathrm{~m}, 1 \mathrm{H}, 3 \mathrm{~b}-\mathrm{H})$, $1.97(\mathrm{~m}, 1 \mathrm{H}, 3 \mathrm{a}-\mathrm{H}), 1.90(\mathrm{~m}, 1 \mathrm{H}, 2 \mathrm{~b}-\mathrm{H}) ;{ }^{13} \mathrm{C}$ NMR data $\left(\mathrm{D}_{2} \mathrm{O}, 125 \mathrm{MHz}\right) \delta_{\mathrm{C}} 164.9(\mathrm{C}-5), 158.8(\mathrm{C}-11), 158.0(\mathrm{C}-14)$, 133.7 (C-10), 125.8 (C-9), 66.4 (C-1), 61.0 (C-4), 46.7 (C-7), 30.7 (C-2), 29.6 (C-8), 28.7 (C-3) ; ${ }^{1} \mathrm{H}$ NMR data (DMSO-d6, 500MHz) $\delta_{\mathrm{H}} 7.91$ (brs, $\left.1 \mathrm{H}, 13-\mathrm{NH}\right), 6.13(\mathrm{t}, J=3 \mathrm{~Hz}, 1 \mathrm{H}, 9-\mathrm{H}), 5.64(\mathrm{dd}, J=3.7$ and $7.3 \mathrm{~Hz}, 1 \mathrm{H}, 1-\mathrm{H}$ ), $4.46(\mathrm{dd}, J=7.2$ and $8.9 \mathrm{~Hz}, 1 \mathrm{H}, 4-\mathrm{H}), 3.92-3,80(\mathrm{~m}, 2 \mathrm{H}, 7 \mathrm{a}-\mathrm{H}$ and $7 \mathrm{~b}-\mathrm{H}), 2.72(\mathrm{~m}, 2 \mathrm{H}, 8 \mathrm{a}-\mathrm{H}$ and 8b-H), $2.32(\mathrm{~m}$, $2 \mathrm{H}, 2 \mathrm{a}-\mathrm{H}$ and $3 \mathrm{~b}-\mathrm{H}$ ), 1.87 (m, 2H, 3a-H and 2b-H); ${ }^{33} \mathrm{C}$ NMR data (DMSO-d6, 125MHz) $\delta_{\mathrm{C}} 161.4$ (C-5), 157.3 (C-14), 155.7 (C-11), 133.9 (C-10), 119.7 (C-9), 64.6 (C-1), 59.4 (C-4), 44.9 (C-7), 29.2 (C-2), 28.0 (C-8), 27.8 (C-3) ; IR (neat) 3392, 1666, 1634, $1595 \mathrm{~cm}^{-1}$; HRMS m/z 250.1296 (250.1304 calcd. for $\mathrm{C}_{11} \mathrm{H}_{16} \mathrm{~N}_{5} \mathrm{O}_{2}, \mathrm{M}+\mathrm{H}$ ). 


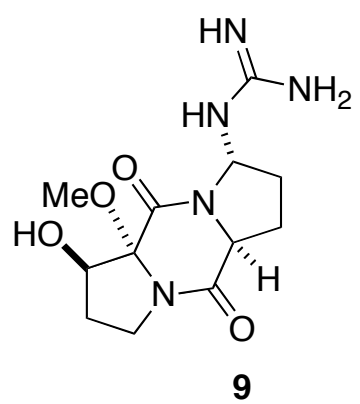

Verpacamide D (as formic acid salt) (9) : colorless solid; ${ }^{1} \mathrm{H} N \mathrm{NR}$ data $\left(\mathrm{CD}_{3} \mathrm{OD}, 600 \mathrm{MHz}\right) \delta_{\mathrm{H}} 5.76(\mathrm{dd}, \mathrm{J}=$ 5.2 and $7.2 \mathrm{~Hz}, 1 \mathrm{H}, 1-\mathrm{H}), 4.66(\mathrm{dd}, J=7.2$ and $9.9 \mathrm{~Hz}, 1 \mathrm{H}, 4-\mathrm{H}), 4.40(\mathrm{~d}, J=4.0 \mathrm{~Hz}, 1 \mathrm{H}, 9-\mathrm{H}), 3.89(\mathrm{~m}, 1 \mathrm{H}, 7 \mathrm{a}-\mathrm{H})$, $3.48(\mathrm{~m}, 1 \mathrm{H}, 7 \mathrm{~b}-\mathrm{H}), 3.30$ (under $\mathrm{MeOH}, \mathrm{Me}), 2.52(\mathrm{~m}, 1 \mathrm{H}, 2 \mathrm{a}-\mathrm{H}), 2.50(\mathrm{~m}, 1 \mathrm{H}, 3 \mathrm{~b}-\mathrm{H}), 2.27(\mathrm{~m}, 1 \mathrm{H}, 8 \mathrm{~b}-\mathrm{H}), 2.03(\mathrm{~m}$, $1 \mathrm{H}, 3 \mathrm{a}-\mathrm{H}), 1.93(\mathrm{~m}, 2 \mathrm{H}, 2 \mathrm{~b}-\mathrm{H}$ and $8 \mathrm{a}-\mathrm{H}) ;{ }^{13} \mathrm{C}$ NMR data deduced from the $2 \mathrm{D}\left({ }^{1} \mathrm{H}-{ }^{13} \mathrm{C}\right)$ spectra $\left(\mathrm{CD}_{3} \mathrm{OD}\right.$, $150 \mathrm{MHz}) \delta_{\mathrm{C}} 168.8$ (C-5), 158.8 (C-14), 97.0 (C-10), 75.1 (C-9), 66.9 (C-1), 60.1 (C-4), 44.2 (C-7), 30.7 (C-2), 29.3 (C-8), 28.5 (C-3) ; ${ }^{1} \mathrm{H}$ NMR data (DMSO-d6, 800MHz) $\delta_{\mathrm{H}} 8.19$ (d, J = 8.0 Hz, 1H, 13-NH), 5.67 (m, 1H, 1-H), 5.38 (d, $J=4.5 \mathrm{~Hz}, 1 \mathrm{H}, \mathrm{OH}), 4.65$ (brt, 1H, 4-H), $4.18(\mathrm{~m}, 1 \mathrm{H}, 9-\mathrm{H}), 3.80(\mathrm{~m}, 1 \mathrm{H}, 7 \mathrm{a}-\mathrm{H}), 3.30$ (under $\left.\mathrm{H}_{2} \mathrm{O}, 7 \mathrm{~b}-\mathrm{H}\right), 3.19(\mathrm{~s}$, $3 \mathrm{H}, \mathrm{Me}), 2.37(\mathrm{~m}, 1 \mathrm{H}, 2 \mathrm{a}-\mathrm{H}), 2.33(\mathrm{~m}, 1 \mathrm{H}, 3 \mathrm{~b}-\mathrm{H}), 2.07(\mathrm{~m}, 2 \mathrm{H}, 8 \mathrm{~b}-\mathrm{H}), 1.80(\mathrm{~m}, 1 \mathrm{H}, 2 \mathrm{~b}-\mathrm{H}), 1.76(\mathrm{~m}, 1 \mathrm{H}, 8 \mathrm{a}-\mathrm{H}), 1.75$ (m, 1H, 3a-H) ; HRMS m/z 298.1496 (298.1515 calcd. for $\left.\mathrm{C}_{12} \mathrm{H}_{20} \mathrm{~N}_{5} \mathrm{O}_{4}, \mathrm{M}+\mathrm{H}\right)$. 


\section{Spectra:}

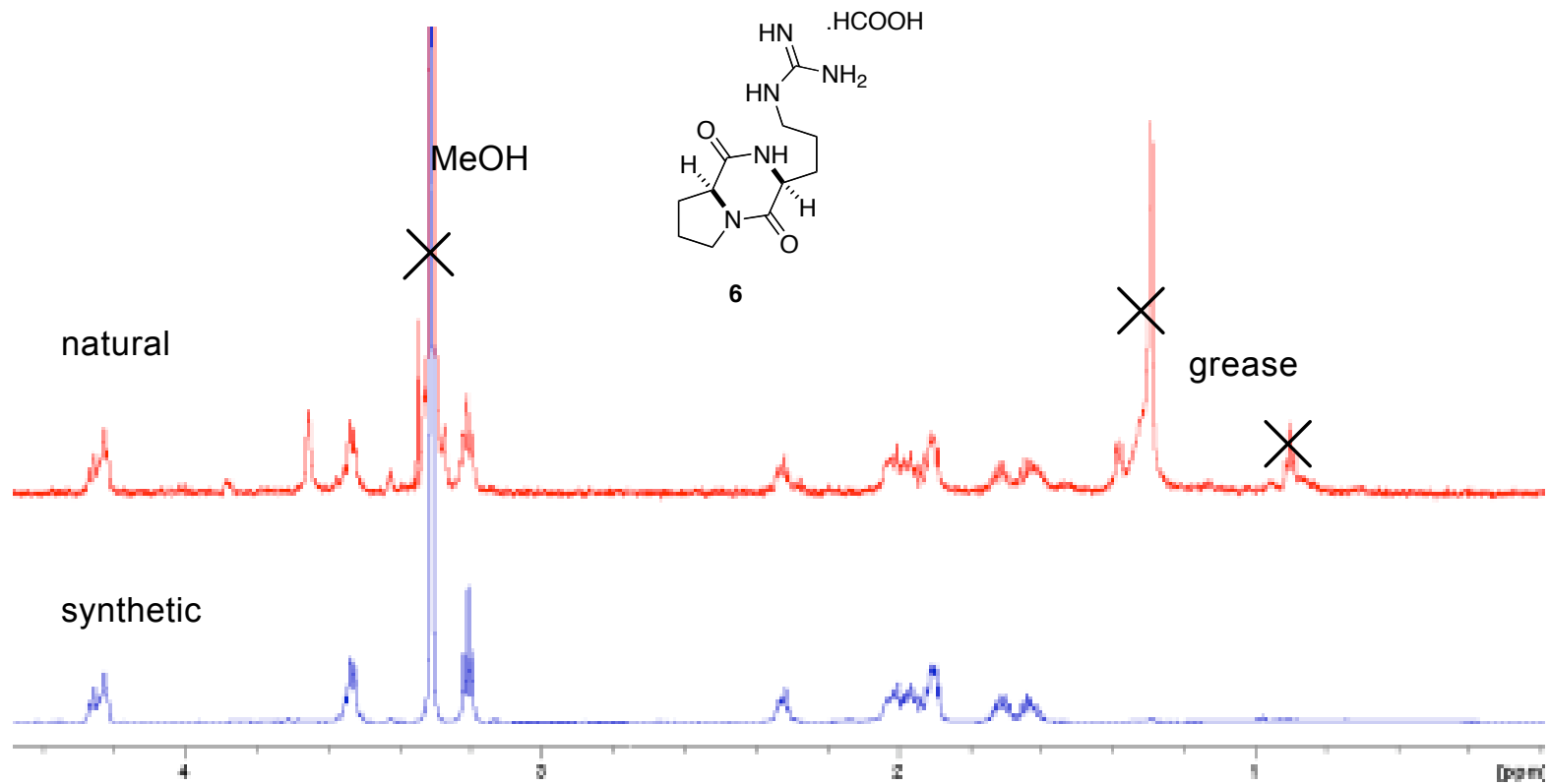

Figure S1. ${ }^{1} \mathrm{H}$ NMR spectra of natural and synthetic 6 in $\mathrm{CD}_{3} \mathrm{OD}(600 \mathrm{MHz})$

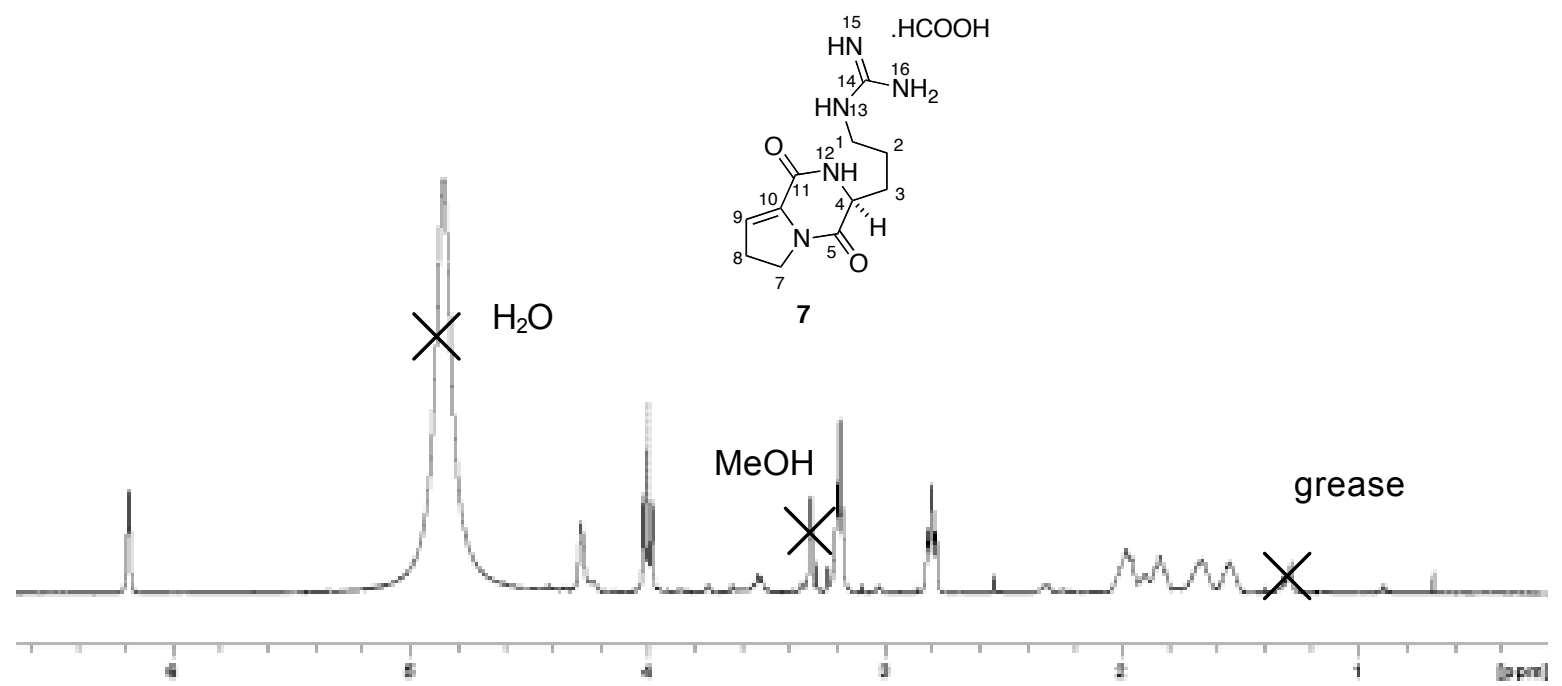

Figure S2. ${ }^{1} \mathrm{H}$ NMR spectrum of 7 in $\mathrm{CD}_{3} \mathrm{OD}(500 \mathrm{MHz})$ 


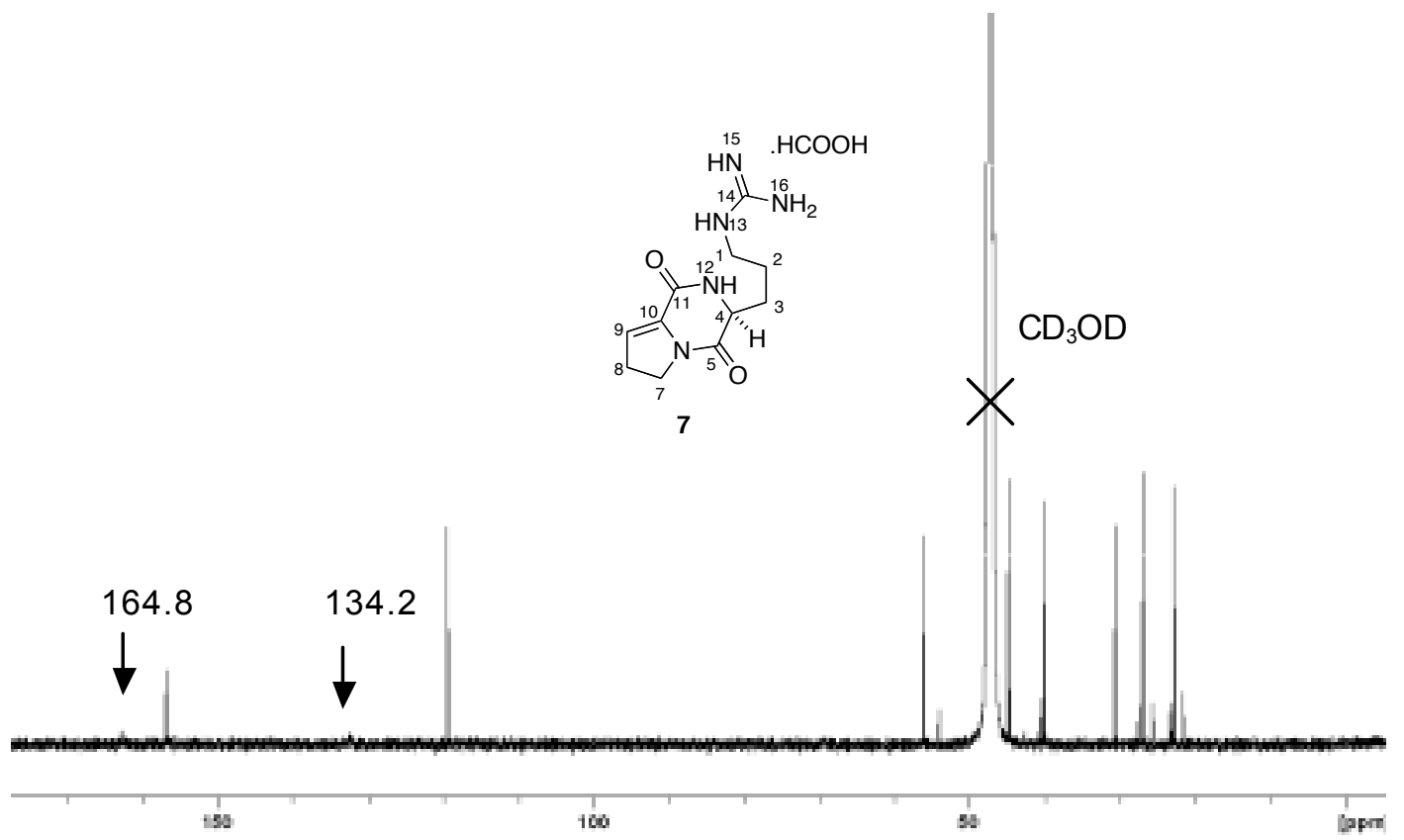

Figure S3. ${ }^{13} \mathrm{C}$ NMR spectrum of 7 in $\mathrm{CD}_{3} \mathrm{OD}(500 \mathrm{MHz})$

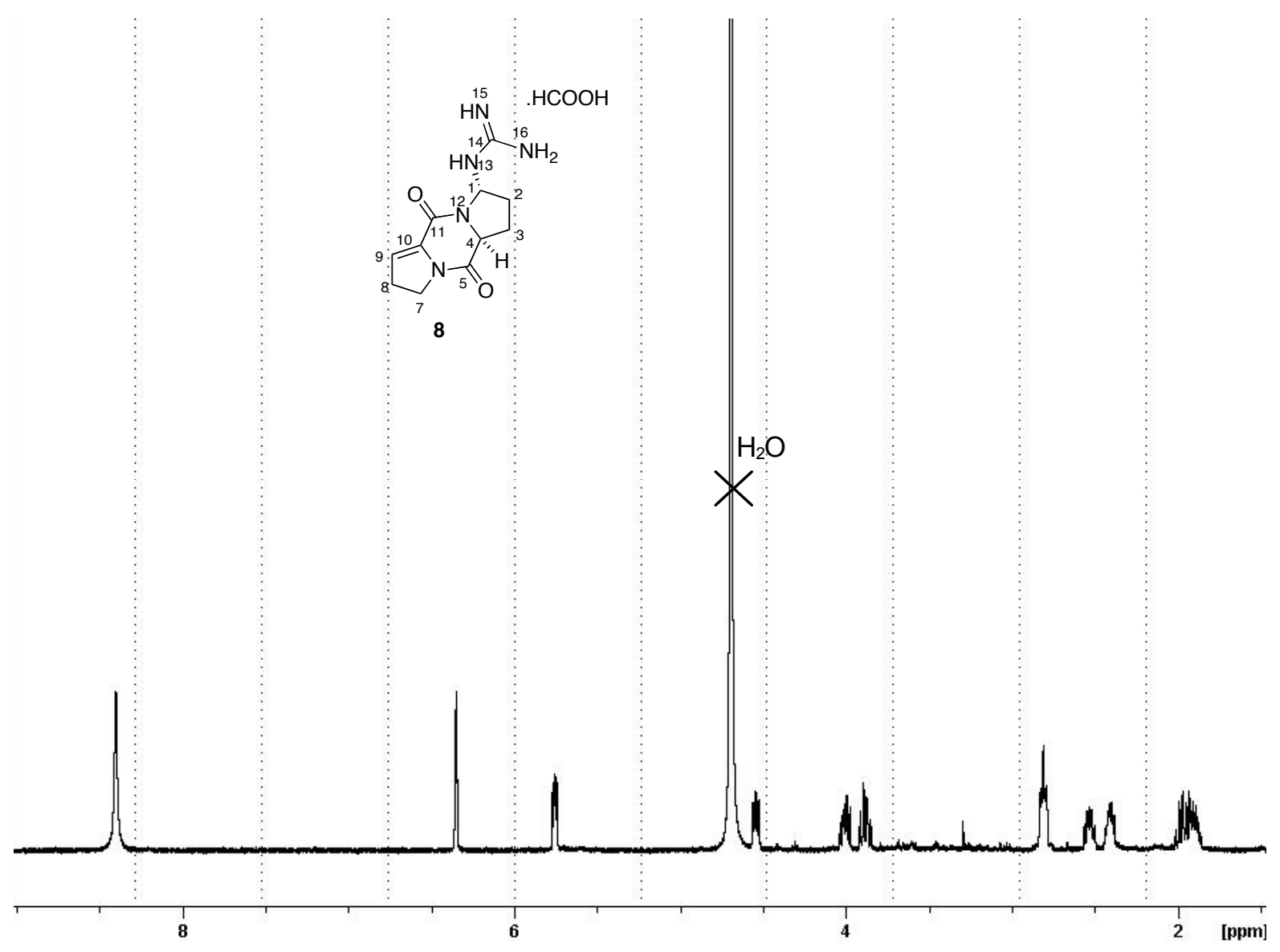


Figure S4. ${ }^{1} \mathrm{H}$ NMR spectrum of 8 in $\mathrm{D}_{2} \mathrm{O}(500 \mathrm{MHz})$

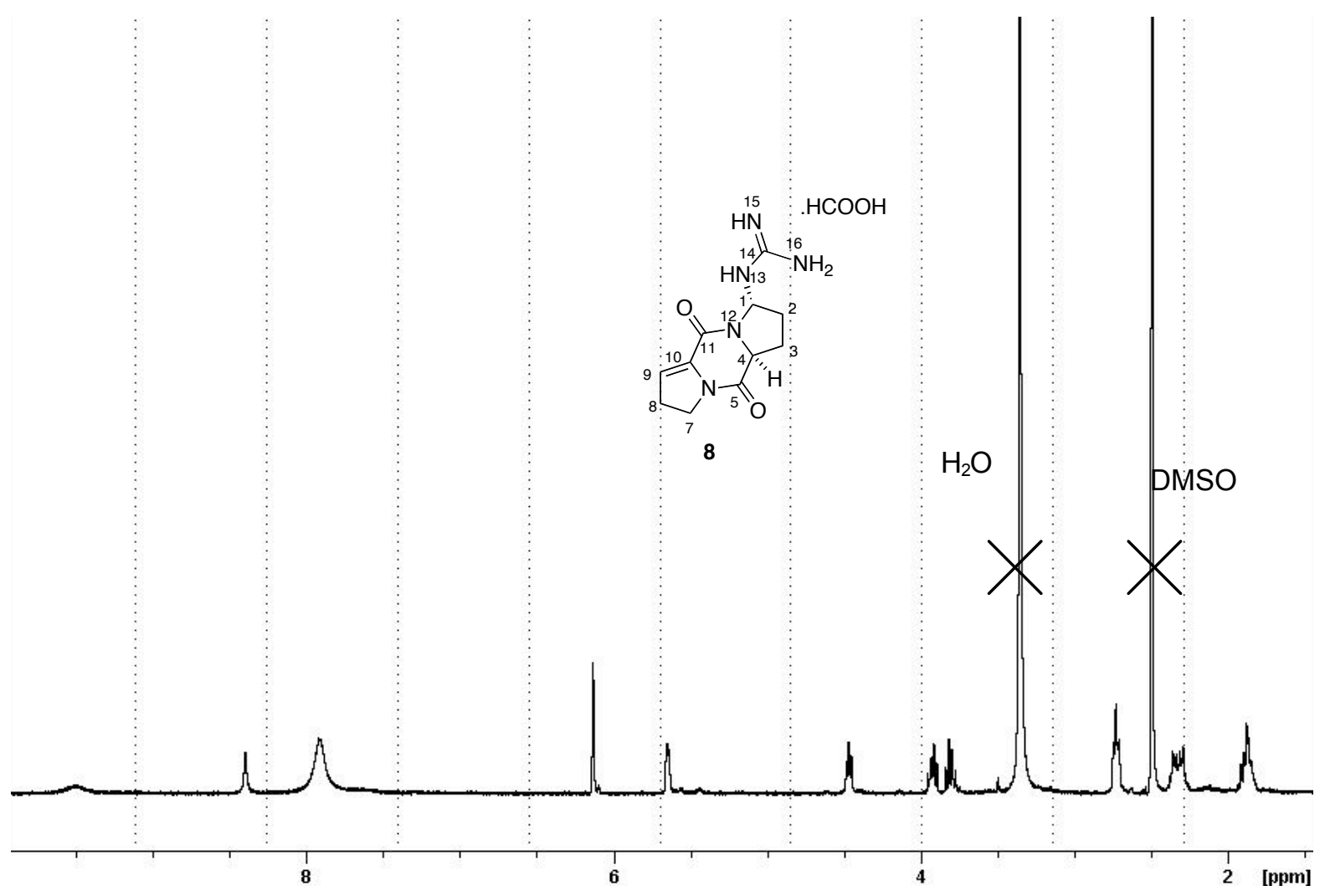

Figure S5. ${ }^{1} \mathrm{H}$ NMR spectrum of 8 in DMSO-d6 $(500 \mathrm{MHz})$
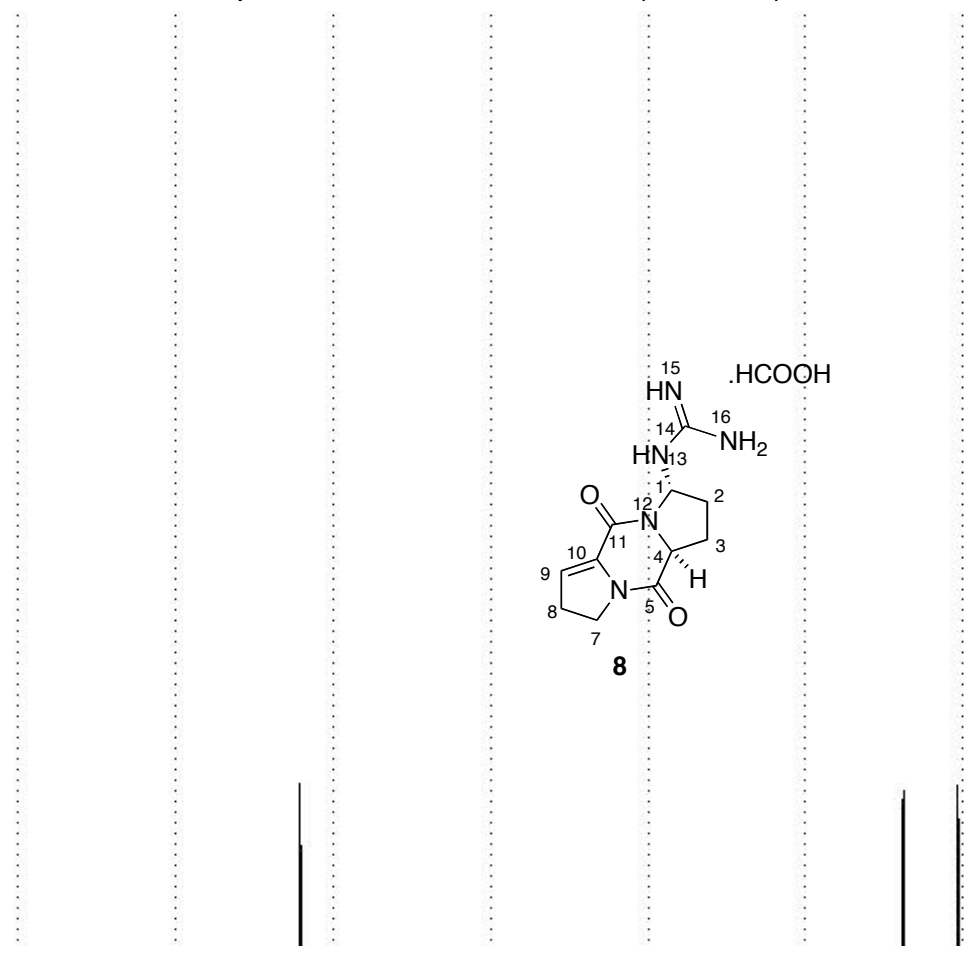
Figure S6. ${ }^{13} \mathrm{C}$ NMR spectrum of 8 in $\mathrm{D}_{2} \mathrm{O}(500 \mathrm{MHz})$

Figure S6. ${ }^{13} \mathrm{C}$ NMR spectrum of 8 in $\mathrm{D}_{2} \mathrm{O}(500 \mathrm{MHz})$

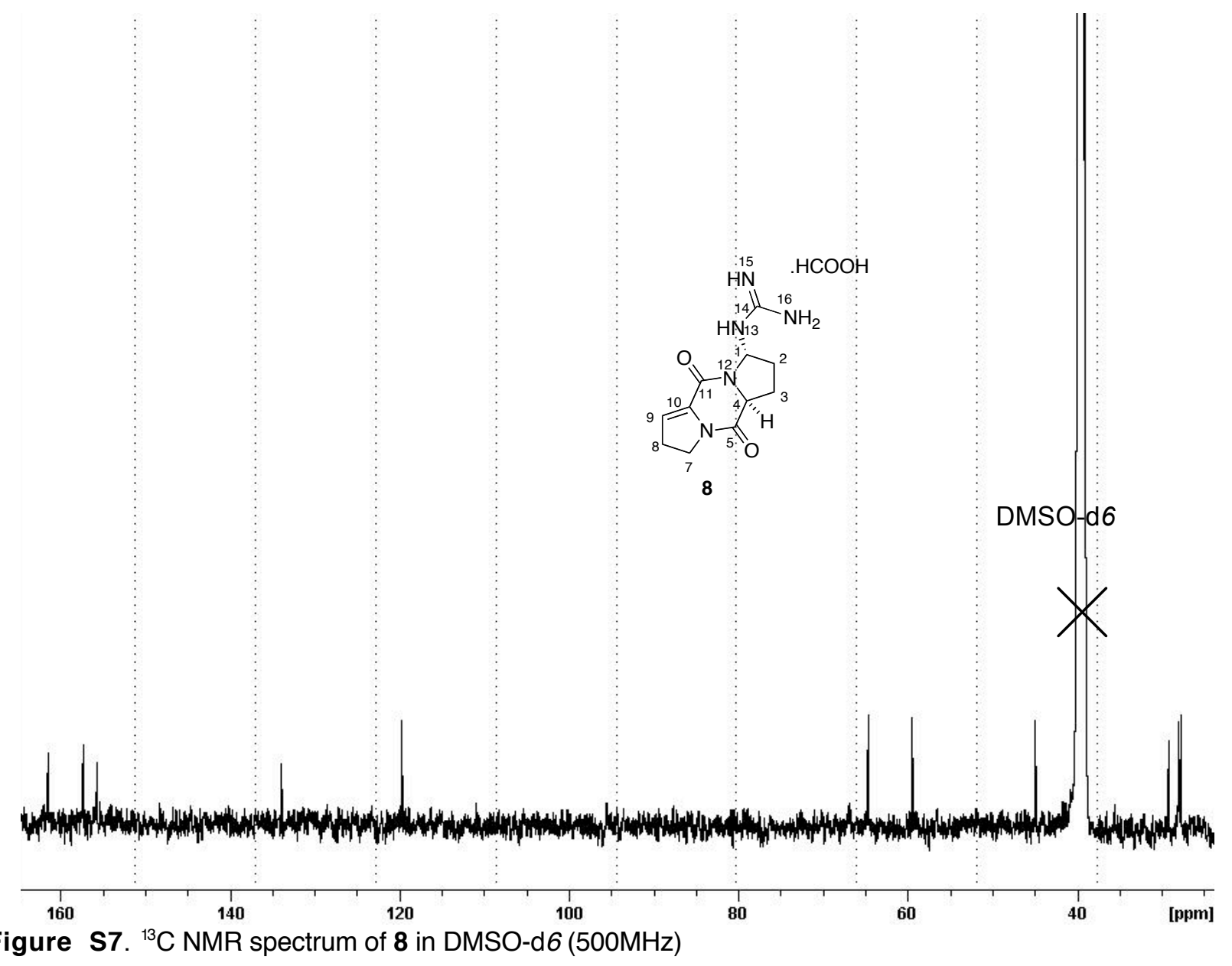

Figure S7. ${ }^{13} \mathrm{C}$ NMR spectrum of 8 in DMSO-d6 $(500 \mathrm{MHz})$

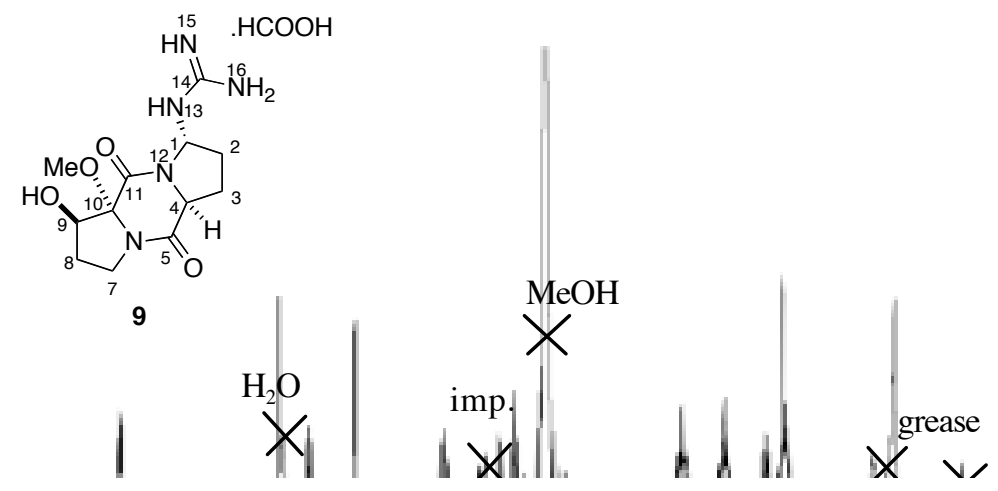


Figure S8. ${ }^{1} \mathrm{H}$ NMR spectrum of 9 in $\mathrm{CD}_{3} \mathrm{OD}(600 \mathrm{MHz})$ 